\title{
On the ground state energy scaling in quasi-rung-dimerized spin ladders
}

\author{
P. N. Bibikov ${ }^{1}$ \\ ${ }^{1}$ Saint-Petersburg State University
}

(Dated: June 25, 2018)

\begin{abstract}
On the basis of periodic boundary conditions we study perturbatively a large $N$ asymptotics $(N$ is the number of rungs) for the ground state energy density and gas parameter of a spin ladder with slightly destroyed rung-dimerization. Exactly rung-dimerized spin ladder is treated as the reference model. Explicit perturbative formulas are obtained for three special classes of spin ladders.

PACS numbers:
\end{abstract}

\section{INTRODUCTION}

Phase structure of frustrated spin ladders and spin ladders with four-spin terms has been intensively studied in the last decade both theoretically and numerically $\underline{\underline{1}}-\underline{\underline{3}}$. Among other phases the mathematically most simple one and at the same time, probably, the one most interesting for physical applications is the so called rung-singlet (or rung-dimerized) phase $e^{4,5}$. Within it the ground state may be well approximated by an infinite tensor product of rung-dimers (singlet pairs)

$$
|0\rangle_{r-d}=\otimes_{n}|0\rangle_{n}
$$

This state will be an exact ground state only for rather big antiferromagnetic rung-coupling and under a special condition on the coupling constants 4 . The latter has no physical background and thus there are absolutely no grounds to assume its relevance for real compounds. Nevertheless it is a common opinion that for rather big antiferromagnetic rung coupling a spin ladder should still remain in the rung-singlet phase. This means that all physical properties of such a ladder may be obtained perturbatively on the basis of the "bare" ground state (1) and its excitations. Together with verification by machinery calculations this approach should give a comprehensive description of the rung-singlet phase. A machinery calculation will provide excellent tests for suggested formulas while a perturbative formula will give a right direction for numerical research and interpretation of the obtained data.

Such approach has two main difficulties. First of all a general spin ladder model is non-integrable and although one- and two-magnon states may be readily derived within Bethe Ansatz, three-magnon states are obtained now only for five special integrable models 6.7 . The second difficulty originates from the fact that an analytical result is usually obtained for infinite ladder however in a numerical calculation a ladder has a finite size. Hence in order to use a mashinery calculation for verification of an analytical result one havs to perform a correct extrapolation of the numerical data. This means that utilizing a finite number of numerical estimations $f_{N}$ of some value $f$ ( $N$ the number rungs of the ladder) it is nesessary to estimate the limit $f_{\infty}=\lim _{N \rightarrow \infty} f_{N}$. On this way, in addition to a number of sequence transformation meth- ods improving the convergence ${ }^{8}$, one has to be guided by some extrapolation formula. The latter may be guessed by an analysis of numerical data ${ }^{9}$, or suggested theoreticallly on the basis of conformal field theory ${ }^{10}$ predictions, or on some other argumentation 11 .

Taking an exact rung-dimerized spin ladder as a reference model, it is natural to treat the ground state of a spin ladder with violated rung-dimerization as a dilute magnon gas ${ }^{12}$. Its consentration (gas parameter)

$$
\rho \equiv \rho_{\infty}=\lim _{N \rightarrow \infty} \rho_{N}, \quad \rho_{N}=\frac{\langle 0|\hat{Q}| 0\rangle}{N},
$$

$(\hat{Q}$ is a magnon number operator (13)) and energy density

$$
E \equiv E_{\infty}=\lim _{N \rightarrow \infty} E_{N}, \quad E_{N}=\frac{\langle 0|\hat{H}| 0\rangle}{N},
$$

turns to zero for an exact rung-dimerized spin ladder and hence they should be good governing parameters for a perturbation theory based on the gas approximation. Perturbative expressions for $\rho$ and $E$ were derived in Ref. 12 . In the present paper assuming periodic boundary conditions we obtain in three special cases the corresponding extrapolation formulas for $\rho_{N}$ and $E_{N}$.

The two formulas

$$
\begin{aligned}
& E_{N}=E_{\infty}+(-1)^{N} A \frac{\mathrm{e}^{-N / N_{0}}}{N^{2}}, \\
& E_{N}=E_{\infty}-\frac{A}{N^{2}},
\end{aligned}
$$

( $A$ and $N_{0}$ are free parameters) have already been suggested correspondingly for open ${ }^{13,14}$ and periodic ${ }^{10}$ boundary conditions. The expression (4) was implied ad hoc, while Eq. (5) follows from conformal theory argumentation. The perturbative formulas obtained below for three special classes of spin ladders have a rather different form

$$
E_{N}=E_{\infty}+\left(A+(-1)^{N} B\right) \mathrm{e}^{-(N-1) / N_{0}} .
$$

\section{DESCRIPTION OF THE MODEL}

We shall use an equivalent representation 6.12

$$
\hat{H}=\hat{H}_{0}+J_{6} \hat{V},
$$


of the spin ladder Hamiltonian $\underline{\underline{1}} \underline{\underline{\underline{5}}}$. Here $J_{6}$ is a perturbation parameter and

$$
\begin{aligned}
\hat{H}_{0} & =\sum_{n=1}^{N} J_{1} Q_{n}+J_{2}\left(\mathbf{\Psi}_{n} \cdot \overline{\mathbf{\Psi}}_{n+1}+\overline{\mathbf{\Psi}}_{n} \cdot \mathbf{\Psi}_{n+1}\right) \\
& +J_{3} Q_{n} Q_{n+1}+J_{4} \mathbf{S}_{n} \cdot \mathbf{S}_{n+1}+J_{5}\left(\mathbf{S}_{n} \cdot \mathbf{S}_{n+1}\right)^{2}, \\
\hat{V} & =\sum_{n=1}^{N} V_{n, n+1}, \\
\mathbf{S}_{n} & =\mathbf{S}_{1, n}+\mathbf{S}_{2, n}, \quad Q_{n}=\frac{1}{2} \mathbf{S}_{n}^{2} \\
V_{n, n+1} & =\tilde{\mathbf{\Psi}}_{n} \cdot \overline{\mathbf{\Psi}}_{n+1}+\mathbf{\Psi}_{n} \cdot \mathbf{\Psi}_{n+1},
\end{aligned}
$$

$\left(\mathbf{S}_{i, n}\right.$ for $i=1,2$ are spin-1/2 operators associated with $n$-th rung). The local operators

$$
\begin{aligned}
& \mathbf{\Psi}_{n}=\frac{1}{2}\left(\mathbf{S}_{1, n}-\mathbf{S}_{2, n}\right)-i\left[\mathbf{S}_{1, n} \times \mathbf{S}_{2, n}\right], \\
& \overline{\mathbf{\Psi}}_{n}=\frac{1}{2}\left(\mathbf{S}_{1, n}-\mathbf{S}_{2, n}\right)+i\left[\mathbf{S}_{1, n} \times \mathbf{S}_{2, n}\right],
\end{aligned}
$$

may be interpreted as (neither Bose nor Fermi) creationannihilation operators for rung-triplets. Namely

$$
\begin{aligned}
\overline{\mathbf{\Psi}}_{n}^{a}|0\rangle_{n}=|1\rangle_{n}^{a}, & \overline{\mathbf{\Psi}}_{n}^{a}|1\rangle_{n}^{b}=0, \\
\boldsymbol{\Psi}_{n}^{a}|0\rangle_{n}=0, & \boldsymbol{\Psi}_{n}^{a}|1\rangle_{n}^{b}=\delta_{a b}|0\rangle_{n} .
\end{aligned}
$$

From (8) and (9) readily follows $\frac{6}{6}$ that

$$
\left[\hat{H}_{0}, \hat{Q}\right]=0 \text {, }
$$

where the operator

$$
\hat{Q}=\sum_{n} Q_{n}
$$

according to relations

$$
Q_{n}|0\rangle=0, \quad Q_{m}|1\rangle_{n}=\delta_{m n}|1\rangle_{n},
$$

has a sence of the number operator for rung-triplets 6 .

For rather big $J_{1}$ (for example nesessary should be ${ }^{4,6}$ $\left.J_{1}>J_{2}\right)$ vector (1) is the zero energy $\left(\hat{H}_{0}|0\rangle_{r-d}=0\right)$ ground state for $\hat{H}_{0}$, whose physical Hilbert space splits into a direct sum $4,6,12$

$$
\mathcal{H}=\sum_{m=0}^{\infty} \mathcal{H}^{m},\left.\quad \hat{Q}\right|_{\mathcal{H}^{m}}=m .
$$

The subspace $\mathcal{H}^{0}$ is generated by the single vector (1). According to (2), (3) and (8)

$$
\rho_{N}=\frac{\partial E_{N}}{\partial J_{1}}
$$

Since $\hat{V}:|0\rangle_{r-d} \rightarrow \mathcal{H}^{2}$, a perturbative treatment of the term $J_{6} \hat{V}$ gives

$$
E_{N}=-\frac{J_{6}^{2}}{N} \sum_{|\mu\rangle \in \mathcal{H}^{2}} \frac{\left|\langle\mu|\hat{V}| 0\rangle_{r-d}\right|^{2}}{E(\mu)}+o\left(J_{6}^{2}\right),
$$

where all the states $|\mu\rangle$ in the sum have zero total spin and quasimomentum. In the $N \rightarrow \infty$ limit 12

$$
\begin{gathered}
E_{\infty}=-\Theta\left(\Delta_{0}^{2}-1\right) \frac{3 J_{6}^{2}\left(\Delta_{0}^{2}-1\right)}{\Delta_{0}^{2} E_{\text {bound }}} \\
-\frac{3 J_{6}^{2}}{4 J_{2} \Delta_{0}}\left(1-\frac{J_{2}\left|\Delta_{0}^{2}-1\right|+2 \Delta_{0} \sqrt{J_{1}^{2}-J_{2}^{2}}}{\left[2 \Delta_{0} J_{1}+\left(\Delta_{0}^{2}+1\right) J_{2}\right]}\right)
\end{gathered}
$$

where $\Theta(x)=1$ for $x>0$ and $\Theta(x)=0$ for $x \leq 0$ and

$$
\begin{aligned}
\Delta_{0} & =\frac{J_{3}-2 J_{4}+4 J_{5}}{2 J_{2}} \\
E_{\text {bound }} & =4 J_{1}+2 J_{2}\left(\Delta_{0}+\frac{1}{\Delta_{0}}\right) .
\end{aligned}
$$

\section{A FINITE- $N$ TWO-PARTICLE PROBLEM}

A zero total spin and quasimomentum two-magnon state has the following general form,

$$
|2-\operatorname{magn}\rangle=\sum_{1 \leq m<n \leq N} a(n-m) \ldots|1\rangle_{m}^{a} \ldots|1\rangle_{n}^{a} \ldots
$$

The dimension of the corresponding Hilbert space is $N / 2$ for even $N$ and $(N-1) / 2$ for odd. The wave function $a(n)$ should be normalised

$$
\sum_{n=1}^{N-1}(N-n)|a(n)|^{2}=\sum_{m<n}|a(n-m)|^{2}=\frac{1}{3},
$$

and satisfy the periodicity condition $a(n-m)=a(m+$ $N-n$ ) or shortly

$$
a(n)=a(N-n) .
$$

Performing a substitution $n \rightarrow N-n$ and using (23) one can obtain from (22)

$$
\sum_{n=1}^{N-1} n|a(N-n)|^{2}=\sum_{n=1}^{N-1} n|a(n)|^{2}=\frac{1}{3} .
$$

Together (22) and (24) result in

$$
\sum_{n=1}^{N-1}|a(n)|^{2}=\frac{2}{3 N} .
$$

The Schrödinger equation gives

$$
4 J_{1} a(n)+2 J_{2}[a(n-1)+a(n+1)]=E a(n)
$$

for $1<n<N-1$ and

$$
2\left(2 J_{1}+J_{2} \Delta_{0}\right) a(1)+2 J_{2} a(2)=E a(1),
$$

for $n=1$. 
General solution of the system (26), (27) has the form

$$
a(n, z)=\frac{1}{\sqrt{Z(z)}}\left[\left(1-\frac{\Delta_{0}}{z}\right) z^{n}-\frac{1}{z^{n}}\left(1-\Delta_{0} z\right)\right],
$$

and dispersion

$$
E(z)=4 J_{1}+2 J_{2}\left(z+\frac{1}{z}\right)
$$

The normalization constant $Z(z)$ ensures condition (25). The parameter $z$ corresponds to relative quasimomentum of magnon pair and satisfy an equation

$$
z^{N-1}=\frac{\Delta_{0} z-1}{z-\Delta_{0}}=-z \frac{\Delta_{0}-1 / z}{\Delta_{0}-z} .
$$

The latter is invariant under complex conjugation and a duality symmetry

$$
z \rightarrow \frac{1}{z}
$$

which according to (28) is related to multiplication of the wave function on $(-1)$. Hence for even $N$ the roots of (30) are joined in dual pairs, while for odd $N$ there is an additional autodual root $z=-1$.

In the three special cases $\Delta_{0}=-1, \Delta_{0}=1$ and $\Delta_{0}=0$ Eq. (30) may be solved explicitly. Denoting the corresponding solutions as $u_{j}, v_{j}$ and $w_{j}$ respectively one has
Taking into account that all the roots (32) lie in a unite circle one may readily get

$$
\begin{aligned}
& Z(z)=3 N(N-1)\left(1-\Delta_{0} z\right)\left(1-\frac{\Delta_{0}}{z}\right), \quad \Delta_{0}= \pm 1 \\
& Z(z)=3 N^{2}, \quad \Delta=0
\end{aligned}
$$

and then

$$
\begin{aligned}
|a(n, z)|^{2}= & \frac{1}{3 N(N-1)}\left[2+\Delta_{0}\left(z^{2 n-1}+\frac{1}{z^{2 n-1}}\right)\right] \\
& \Delta_{0}= \pm 1 \\
|a(n, z)|^{2}= & \frac{1}{3 N^{2}}\left(2-z^{2 n}-\frac{1}{z^{2 n}}\right), \quad \Delta_{0}=0
\end{aligned}
$$

\section{EXACT RESULTS AT $\Delta_{0}=0$ AND $\Delta_{0}= \pm 1$}

Let $|z\rangle$ be the state related to wave function (28). From (9) and (21) follows that

$$
\left|\langle z|\hat{V}| 0\rangle_{r-d}\right|^{2}=9 N^{2}|a(1, z)|^{2} .
$$

For the evaluation of $E_{N}$ one has to perform in (17) a summation over all duality pairs of roots. Since both the roots in a pair give the same contribution this is equivalent to inserting the factor $1 / 2$ before summation over all roots. Hence (17) and (35) result in

$$
E_{N}\left(\Delta_{0}\right)=-\frac{3}{4} J_{6}^{2} G_{N}\left(\Delta_{0}\right)+o\left(J_{6}^{2}\right),
$$

where

$$
\begin{aligned}
& G_{N}(-1)=\frac{1}{N-1} \sum_{j=0}^{N-2} \frac{2-\left(u_{j}+1 / u_{j}\right)}{2 J_{1}+J_{2}\left(u_{j}+1 / u_{j}\right)}=\frac{1}{J_{2}(N-1)} \sum_{j=0}^{N-2}\left[-1+\frac{J_{1}+J_{2}}{\sqrt{J_{1}^{2}-J_{2}^{2}}}\left(\frac{J_{-}}{J_{-}-u_{j}}-\frac{J_{+}}{J_{+}-u_{j}}\right)\right] \\
& =\frac{1}{J_{2}}\left[\frac{J_{1}+J_{2}}{\sqrt{J_{1}^{2}-J_{2}^{2}}}\left(\frac{J_{-}^{N-1}}{J_{-}^{N-1}+1}-\frac{J_{+}^{N-1}}{J_{+}^{N-1}+1}\right)-1\right] \\
& G_{N}(1)=\frac{1}{N-1} \sum_{j=0}^{N-2} \frac{2+\left(v_{j}+1 / v_{j}\right)}{2 J_{1}+J_{2}\left(v_{j}+1 / v_{j}\right)}=\frac{1}{J_{2}(N-1)} \sum_{j=0}^{N-2}\left[1-\frac{J_{1}-J_{2}}{\sqrt{J_{1}^{2}-J_{2}^{2}}}\left(\frac{J_{-}}{J_{-}-v_{j}}-\frac{J_{+}}{J_{+}-v_{j}}\right)\right], \\
& =\frac{1}{J_{2}}\left[1-\frac{J_{1}-J_{2}}{\sqrt{J_{1}^{2}-J_{2}^{2}}}\left(\frac{J_{-}^{N-1}}{J_{-}^{N-1}+(-1)^{N-1}}-\frac{J_{+}^{N-1}}{J_{+}^{N-1}+(-1)^{N-1}}\right)\right] \text {, } \\
& G_{N}(0)=\frac{1}{N} \sum_{j=0}^{N-1} \frac{2 w_{j}^{2}-w_{j}^{4}-1}{w_{j}\left(J_{2} w_{j}^{2}-2 J_{1} w_{j}+J_{2}\right)}=\frac{2}{J_{2}^{2} N} \sum_{j=0}^{N-1}\left[J_{1}-\frac{J_{2}}{2}\left(w_{j}+\frac{1}{w_{j}}\right)-\sqrt{J_{1}^{2}-J_{2}^{2}}\left(\frac{J_{-}}{J_{-}-w_{j}}-\frac{J_{+}}{J_{+}-w_{j}}\right)\right] \\
& =2\left[\frac{J_{1}}{J_{2}^{2}}-\frac{\sqrt{J_{1}^{2}-J_{2}^{2}}}{J_{2}^{2}}\left(\frac{J_{-}^{N}}{J_{-}^{N}+1}-\frac{J_{+}^{N}}{J_{+}^{N}+1}\right)\right]
\end{aligned}
$$

and

$$
J_{ \pm}=\frac{-J_{1} \pm \sqrt{J_{1}^{2}-J_{2}^{2}}}{J_{2}}
$$

In (37) we used for calculations the formulas

$$
\begin{aligned}
& \sum_{j=0}^{N-2} \frac{1}{J-u_{j}}=\frac{(N-1) J^{N-2}}{J^{N-1}+1} \\
& \sum^{N-2} \frac{1}{J-\nu}=\frac{(N-1) J^{N-2}}{I N-1+(-1) N}
\end{aligned}
$$


which may be proved according to the following argumentation. The sums in (39) are fractions whose numerator and denominator are symmetric polynomials with respect to $u_{j}, v_{j}$ and $w_{j}$ respectively. However according to (30) all these polynomials exept

$$
\begin{aligned}
u_{0} \ldots u_{N-2} & =(-1)^{N-1}, \quad v_{0} \ldots v_{N-2}=1, \\
w_{0} \ldots w_{N-1} & =(-1)^{N}
\end{aligned}
$$

are equal to zero.

From equality $J_{+} J_{-}=1$ readily follows

$$
\begin{array}{r}
\frac{J_{-}^{N-1}}{J_{-}^{N-1}+1}-\frac{J_{+}^{N-1}}{J_{+}^{N-1}+1}=\frac{1-J_{+}^{N-1}}{1+J_{+}^{N-1}} \\
\frac{J_{-}^{N-1}}{J_{-}^{N-1}+(-1)^{N-1}}-\frac{J_{+}^{N-1}}{J_{+}^{N-1}+(-1)^{N-1}} \\
=\frac{1-\left(-J_{+}\right)^{N-1}}{1+\left(-J_{+}\right)^{N-1}} .
\end{array}
$$

Using (41) one may readily reduce Eqs. (37) to the form

$$
\begin{aligned}
& G_{N}(-1)=\frac{1}{J_{2}}\left[\sqrt{\frac{J_{1}+J_{2}}{J_{1}-J_{2}}} \cdot \frac{1-J_{+}^{N-1}}{1+J_{+}^{N-1}}-1\right], \\
& G_{N}(1)=\frac{1}{J_{2}}\left[1-\sqrt{\frac{J_{1}-J_{2}}{J_{1}+J_{2}}} \cdot \frac{1-\left(-J_{+}\right)^{N-1}}{1+\left(-J_{+}\right)^{N-1}}\right] \text {, } \\
& G_{N}(0)=\frac{2}{J_{2}}\left[\frac{J_{1}}{J_{2}}-\frac{\sqrt{J_{1}^{2}-J_{2}^{2}}}{J_{2}} \cdot \frac{1-J_{+}^{N}}{1+J_{+}^{N}}\right] \text {. }
\end{aligned}
$$

It may be readily observed that the corresponding values for $E_{\infty}\left(\Delta_{0}\right)$ agree with Eq. (18). The scaling law has the form (6) with

$$
\begin{gathered}
A(-1)=0, \quad B(-1)=-\frac{3 J_{6}^{2}}{2 J_{2}} \sqrt{\frac{J_{1}+J_{2}}{J_{1}-J_{2}}}, \\
A(1)=\frac{3 J_{6}^{2}}{2 J_{2}} \sqrt{\frac{J_{1}-J_{2}}{J_{1}+J_{2}}}, \quad B(1)=0, \\
A(0)=0, \quad B(0)=\frac{3 J_{6}^{2}}{J_{2}^{2}} \sqrt{J_{1}^{2}-J_{2}^{2}},
\end{gathered}
$$

at $J_{2}>0$ and

$$
\begin{gathered}
A(-1)=-\frac{3 J_{6}^{2}}{2 J_{2}} \sqrt{\frac{J_{1}+J_{2}}{J_{1}-J_{2}}}, \quad B(-1)=0, \\
A(1)=0, \quad B(1)=-\frac{3 J_{6}^{2}}{2 J_{2}} \sqrt{\frac{J_{1}-J_{2}}{J_{1}+J_{2}}}, \\
A(0)=\frac{3 J_{6}^{2}}{J_{2}^{2}} \sqrt{J_{1}^{2}-J_{2}^{2}}, \quad B(0)=0,
\end{gathered}
$$

at $J_{2}<0$. In both the cases

$$
N_{0}=\frac{1}{\ln \left|J_{2}\right|-\ln \left(J_{1}-\sqrt{J_{1}^{2}-J_{2}^{2}}\right)} .
$$

The corresponding formulas for $\rho_{N}$ have the similar form and may be readily obtained from (16).
1 P. Lecheminant, K. Totsuka, Phys. Rev. B 74, 224426 (2006)

2 M. T. Batchelor, X.-W. Guan, N. Oelkers, Z. Tsuboi, Adv. Phys. 56, (2007), 465.

3 G. Barcza, Ö. Legeza, R. M. Noack, J. Sólyom, Phys. Rev. B 86, 075133 (2012)

4 A. K. Kolezhuk, H.-J. Mikeska Int. J. Mod. Phys. B 12, 2325 (1998)

5 E. Dagotto Rep. Progr. Phys. 62, 1525 (1999)

${ }^{6}$ P. N. Bibikov, J. Phys. A 42, 315212 (2009)

7 P. N. Bibikov, P. P. Kulish, Journ. Math. Sci. 168, 781
(2010)

8 H. Betsuaki, Phys. Rev. B 34, 8125 (1986)

9 J. C. Bonner, M. E. Fisher, Phys. Rev. 135, A640 (1964)

10 K. Hijii, K. Nomura, Phys. Rev. 65, 104413 (2002)

11 K. Buchta, Ö. Legeza, G. Fáth, J. Sólyom, Phys. Rev. B 72, 054433 (2005)

12 P. N. Bibikov, Int. Journ. Mod. Phys. B 25, 1293 (2011)

13 T. Barnes, E. Dagotto, J. Riera, E. S. Swanson, Phys. Rev. B, 47, 3196 (1993)

14 D. B. Yang, W. C. Haxton, Phys. Rev. B 57, 10603 (1998) 